\title{
Low-dose adenosine stress echocardiography: Detection of myocardial viability
}

\author{
Ana Djordjevic-Dikic*, Miodrag Ostojic, Branko Beleslin, Ivana Nedeljkovic, \\ Jelena Stepanovic, Sinisa Stojkovic, Zorica Petrasinovic, Milan Nedeljkovic, \\ Jovica Saponjski and Vojislav Giga
}

\author{
Address: Institute for Cardiovascular Diseases, Dept. for Diagnostic and Catheterization Laboratories, Clinical Center of Serbia, 8 Koste \\ Todorovica, 11000 Belgrade, Yugoslavia \\ Email: Ana Djordjevic-Dikic* - skali@bitsyu.net; Miodrag Ostojic - miodrag.ostojic@kcs.ac.yu; Branko Beleslin - branko.beleslin@kcs.ac.yu; \\ Ivana Nedeljkovic - ivana.nedeljkovic@kcs.ac.yu; Jelena Stepanovic - jelena.stepanovic@kcs.ac.yu; Sinisa Stojkovic - sinisa.stojkovic@kcs.ac.yu; \\ Zorica Petrasinovic - zorica.petrasinovic@kcs.ac.yu; Milan Nedeljkovic - milan.nedeljkovic@kcs.ac.yu; \\ Jovica Saponjski - jovica.saponjski@kcs.ac.yu; Vojislav Giga - giga@eunet.yu \\ * Corresponding author
}

Published: 3 June 2003

Cardiovascular Ultrasound 2003, I:7
Received: 16 April 2003

Accepted: 3 June 2003

This article is available from: http://www.cardiovascularultrasound.com/content/I/I/7

(c) 2003 Djordjevic-Dikic et al; licensee BioMed Central Ltd. This is an Open Access article: verbatim copying and redistribution of this article are permitted in all media for any purpose, provided this notice is preserved along with the article's original URL.

\begin{abstract}
Objective: The aim of this study was to evaluate the diagnostic potential of low-dose adenosine stress echocardiography in detection of myocardial viability.

Background: Vasodilation through low dose dipyridamole infusion may recruit contractile reserve by increasing coronary flow or by increasing levels of endogenous adenosine.

Methods: Forty-three patients with resting dyssynergy, due to previous myocardial infarction, underwent low-dose adenosine $(80,100,110 \mathrm{mcg} / \mathrm{kg} / \mathrm{min}$ in 3 minutes intervals) echocardiography test. Gold standard for myocardial viability was improvement in systolic thickening of dyssinergic segments of $\geq \mathrm{I}$ grade at follow-up. Coronary angiography was done in $4 \mathrm{I}$ pts. Twenty-seven patients were revascularized and 16 were medically treated. Echocardiographic follow up data ( 12 \pm 2 months) were available in 24 revascularized patients.

Results: Wall motion score index improved from rest $1.55 \pm 0.30$ to $1.33 \pm 0.26$ at low-dose adenosine $(p<0.00 \mathrm{I})$. Of the 257 segments with baseline dyssynergy, adenosine echocardiography identified 122 segments as positive for viability, and 135 as necrotic since no improvement of systolic thickening was observed. Follow-up wall motion score index was $I .31 \pm 0.30(p<0.001$ vs. rest). The sensitivity of adenosine echo test for identification of viable segments was $87 \%$, while specificity was $95 \%$, and diagnostic accuracy $90 \%$. Positive and negative predictive values were $97 \%$ and $80 \%$, respectively.
\end{abstract}

Conclusion: Low-dose adenosine stress echocardiography test has high diagnostic potential for detection of myocardial viability in the group of patients with left ventricle dysfunction due to previous myocardial infarction. Low dose adenosine stress echocardiography may be adequate alternative to low-dose dobutamine test for evaluation of myocardial viability. 


\section{Background}

The detection of reversible myocardial dysfunction has clinical and prognostic significance, as recent studies have suggested that patients with nonrevascularized viable myocardium are at increased risk to suffer later cardiac events $[1,2]$, and that dysfunctional but viable segments may recover after revascularization $[3,4]$. In the past decade, promising results have been reported in identifying physiological markers of myocardial viability with 201Tl scintigraphy and PET imaging [5-9]. Echocardiography using low and high doses of dobutamine $[10,11]$ and dipyridamole [12], or both [13], was also successfully introduced as a cost-effective method with comparable diagnostic potential available in everyday clinical practice. The physiologic basis for using pharmacological agents in identification of myocardial viability relies on the demonstration of residual contractile reserve in basally dysfunctional segments. Such reserve can be elicited either by direct stimulation of $\beta 1$ adrenoreceptors with dobutamine, or through a flow mediated increase in contractile function linked to endogenous adenosine accumulation achieved by intravenous infusion of dipyridamole $[14,15]$. The potential advantage of adenosine over dipyridamole, in addition to the extremely short half life $(<10 \mathrm{sec})$ and rapid onset of action, relies on adenosine direct action on A2 receptors, which increases blood flow $[16,17]$, and other possible mechanisms such as replenishment of high energy phosphate stores, decrease in myocardial oxygen consumption, and inhibition of neutrophile action [18-20]. As shown for low dose dipyridamole testing $[12,13]$, lowest adenosine dose eliciting viability, but not provoking ischemia, should be tested for detection of myocardial viability by echocardiography. Therefore, the aim of our study was to evaluate diagnostic value of low dose adenosine stress echocardiography test for identification of myocardial viability.

\section{Methods}

\section{Study Population}

Forty-three patients (34 men and 9 women, mean age 49 \pm 9 ) with history of previous myocardial infarction (more than 3 months before low dose adenosine stress echocardiography test) and resting wall motion dyssynergy were enrolled in the study. Inclusion criteria were: technically satisfactory acoustic window, no history of high degree atrioventricular block, sick sinus syndrome and severe asthmatic or chronic obstructive pulmonary disease. All patients had a previous Q-wave myocardial infarction. Twenty-two patients (51\%) were on antianginal therapy, consisting of nitrates in 21 (49\%), calcium antagonist in $9(20 \%)$, and $\beta$-blockers in $9(20 \%)$ patients. Theophylline, caffeine-containing products and dipyridamole preparations were not allowed for at least $12 \mathrm{~h}$ before the test. The site of myocardial infarction was anteroseptal in 29, inferior in 8 , anterolateral in 3 , inferolateral in 1 , and lateral in 2 patients.

Coronary angiography was performed in 41 patients. Significant coronary artery disease ( $>50 \%$ diameter stenosis) of one vessel was present in 30 patients, of two vessels in 9 patients and of three vessels in 2 patients. Coronary revascularization was performed in 27 (63\%) patients, whereas $16(37 \%)$ patients were medically treated. None of the patients showed clinical, enzymatic, electrocardiographic or echocardiographic evidence of myocardial infarction during revascularization procedure or follow-up period, and all were considered to have had successful revascularization. Echocardiographic follow-up data were available in 24 revascularized patients after $12 \pm 2$ months.

\section{Low Dose Adenosine Stress Echocardiography Protocol}

An infusion line was placed in the right anterocubital vein. Adenosine was administered by incremental infusion doses of 80,100 and $110 \mu \mathrm{g} / \mathrm{kg} / \mathrm{min}$ at 3 minutes intervals (Figure 1). Blood pressure, heart rate and 12-lead electrocardiogram were followed continuously during adenosine infusion, and recorded at the end of each stage. Patients were asked to describe the symptoms they experienced.

\section{Echocardiographic Monitoring and Analysis}

Two-dimensional echocardiography was performed continuously before, during and after adenosine infusion, using a commercially available imaging system (Toshiba SSH60A; Diasonics) with the patient in the left lateral decubitus position. After cessation of the test, echocardiographic imaging was continued until hemodynamic variables or left ventricular wall motion returned to the basal state. All standard echocardiographic views (apical two-, four-, and five-chamber, and parasternal long-axis and

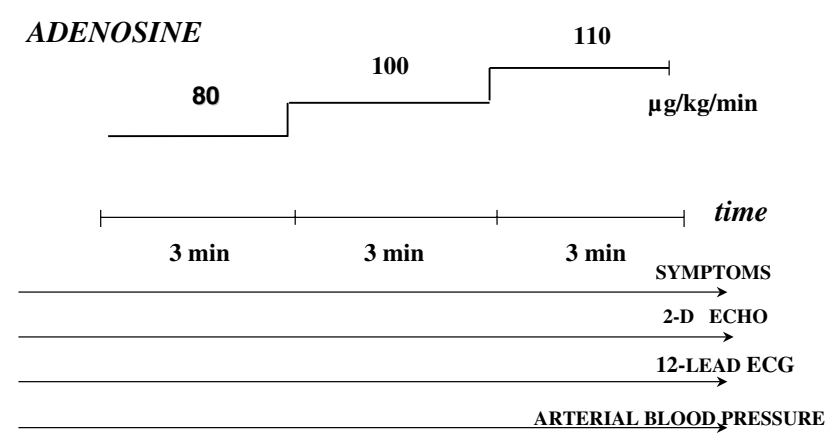

Figure I

Low dose adenosine stress echocardiography study protocol 
short-axis views) were obtained. Echocardiographic images were recorded on VHS videotape for subsequent playback and analysis. An echocardiography test was interpreted from the off-line digitalized videotapes (Image View, ATL) with a side-by-side display of rest and peak stress images in a cine-loop mode. Regional wall motion was assessed according to the recommendations of the American Society of Echocardiography, by a 16-segment model [21]. In all studies, segmental wall motion was semi quantitatively graded as follows: normal $=1$, hypokinetic, marked reduction in endocardial motion = 2, akinetic, virtual absence of inward motion and thickening $=3$, and dyskinetic, paradoxic wall motion away from the center of left ventricle in systole $=4$. A criterion for possible myocardial viability was improvement in systolic thickening of dyssynergic segments of $\geq 1$ grade (hypokinetic segment becoming normal, or akinetic segment becoming hypokinetic), at peak adenosine stress test. The gold standard for viability was improvement in systolic thickening of previously dyssynergic segments of $\geq 1$ grade at resting state at follow-up.

A wall motion score index was calculated for rest and peak stress (9th minute of adenosine infusion) echocardiograms in all patients dividing the sum of individual segment scores by the number of interpreted segments. Inadequately visualized segments were not scored. Echocardiographic follow-up was obtained $\geq 3$ months after adenosine test and/or revascularization procedure. Two experienced observers analyzed all studies separately. Interobserver agreement was $94 \%$ for detection of viability for adenosine testing, and $96 \%$ for identification of viable segments at follow-up.

\section{Statistical Analysis}

Data are expressed as mean value \pm SD. Differences in hemodynamic values and wall motion score index before and at peak adenosine infusion were tested for significance with the Student $t$ test. Dichotomous variables were compared by chi-square (McNemar test for paired proportions). Sensitivity, specificity and diagnostic accuracy were calculated according to the standard definitions. A P value $<0.05$ was considered statistically significant.

\section{Results}

\section{Baseline Echocardiographic Findings}

All patients had a regional dyssynergy in the resting echocardiogram in the territory of previous myocardial infarction. There were 257/688 segments with baseline dyssynergy. The mean resting wall motion score index was $1.55 \pm 0.30$.

\section{Clinical and Hemodynamic Data}

None of the 43 patients had significant side effects. Seven patients $(16 \%)$ complained of flushing. One patient had

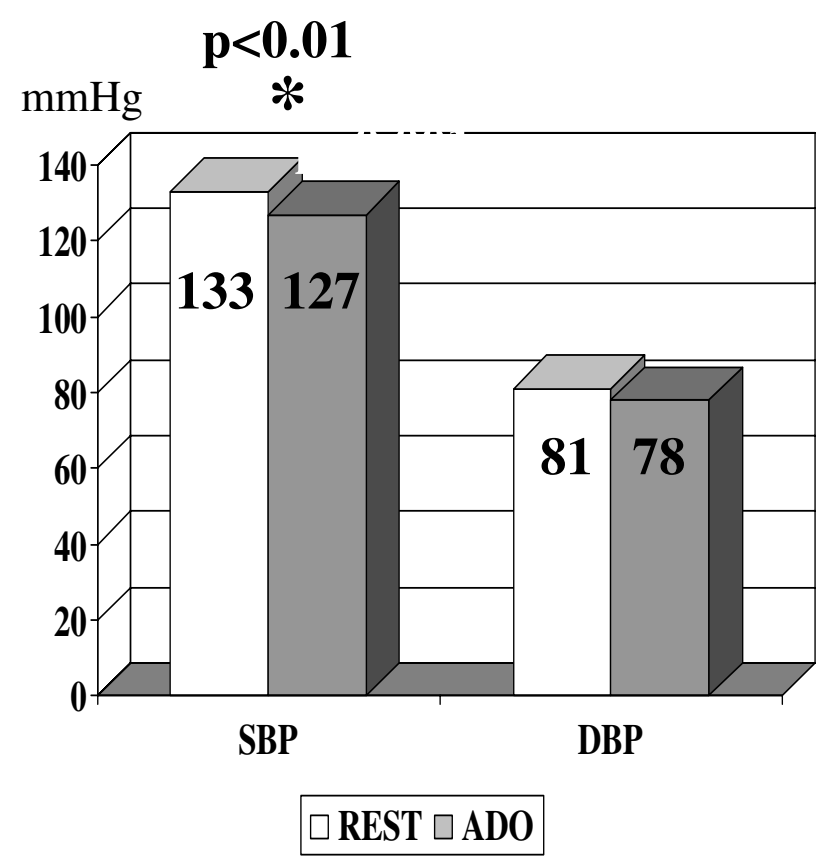

Figure 2

Hemodynamic data during low dose adenosine stress echocardiography test. SBP systolic blood pressure, DBP diastolic blood pressure, ADO adenosine

biphasic response (improvement of function followed by subsequent deterioration) at dose of $110 \mathrm{mcg} / \mathrm{kg} / \mathrm{min}$, with no signs of ischemia on electrocardiogram.

The systemic hemodynamic findings, blood pressure, heart rate, in baseline conditions and during the pharmacological stress tests are shown in Figure 2 and 3. Systolic blood pressure (rest: $133 \pm 15 \mathrm{mmHg}$ ) decreased significantly during the test $(127 \pm 18 \mathrm{mmHg}, \mathrm{p}<0.001 \mathrm{vs}$. rest $)$, while heart rate $(75 \pm 12 \mathrm{bpm}$ at rest) increased to $83 \pm 20$ bpm $(\mathrm{p}<0.001)$.

\section{Basal Echocardiography Findings}

Thirty-one patients (72\%) showed improved segmental wall motion during low dose adenosine stress echocardiography testing, whereas in 12 patients $(28 \%)$ no contractile reserve could be identified. Wall motion score index improved from resting $1.55 \pm 0.30$ to $1.35 \pm 0.28$ ( $\mathrm{p}<$ 0.05 ) at low dose adenosine infusion.

Of the 257 segments with baseline dyssynergy low dose adenosine echocardiography identified 122 segments as viable, and 135 as "necrotic" according to absence of improved thickening and contraction. 


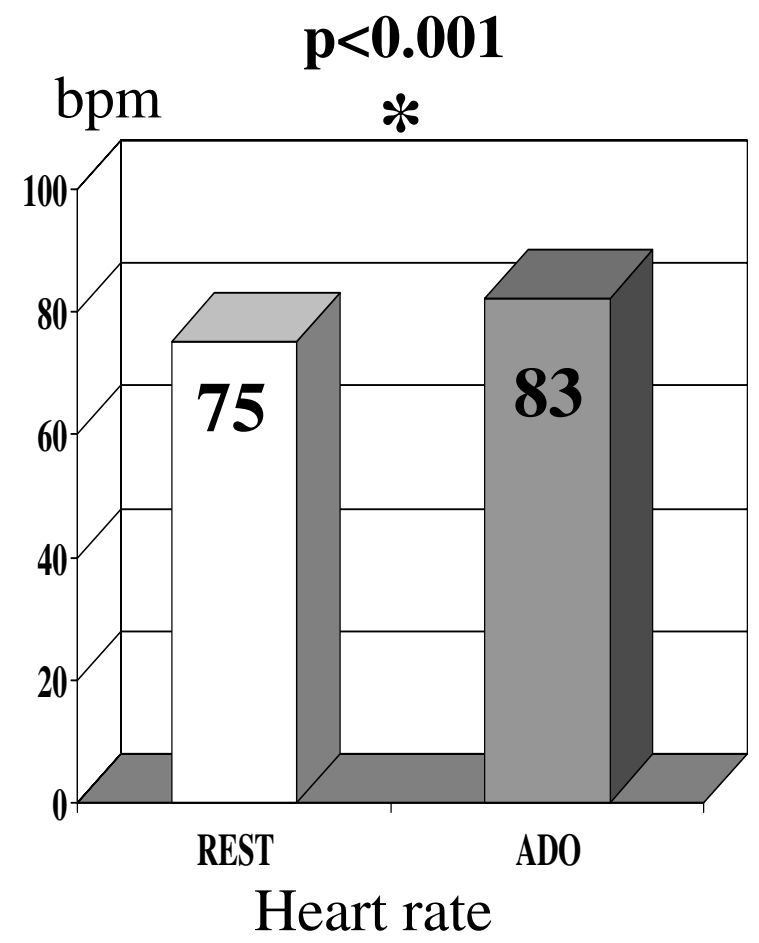

\section{Figure 3}

Hemodynamic data during low dose adenosine stress echocardiography test.

The functional response was not influenced by betablocker therapy.

\section{Diagnostic Potential of Low Dose Adenosine Stress Echocardiography}

Successful coronary revascularization was done in 27 patients; echocardiographic follow-up examination was available in 24 (88\%). Baseline echocardiography, before low dose adenosine testing, showed in these 24 revascularized patients a total of 122 dyssynergic segments. Regional wall motion improved at follow-up by $\geq 1$ grade in 80 segments ("viable", 65\%), whereas in the remaining 42 ("necrotic", 34\%) no improvement could be observed. Of the 80 viable segments, adenosine correctly identified 70 (sensitivity $87 \%$ ). Of the 42 necrotic segments adenosine correctly identified 40 (specificity 95\%). The diagnostic accuracy of adenosine stress echocardiography in predicting the behavior of the basally dyssynergic myocardial segments after revascularization was 90\%. Positive and negative predictive values were $97 \%$ and $80 \%$, respectively (Figure 4). Wall motion score index improved significantly in revascularized patients during follow-up period $(1.54 \pm 0.28$ vs. $1.31 \pm 0.30, \mathrm{p}<0.01)$.

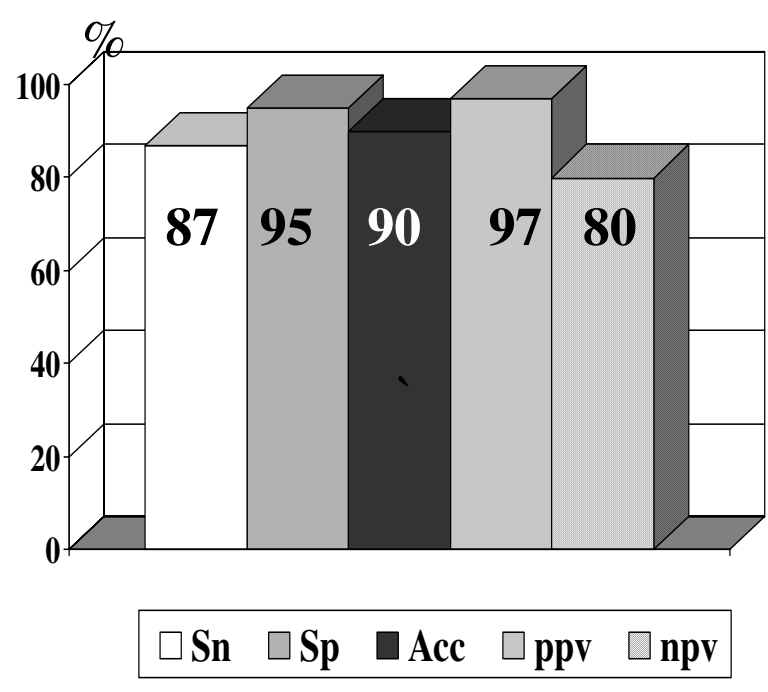

Figure 4

Diagnostic value of low dose adenosine stress echocardiography test in revascularized patients. Sn sensitivity, Sp specificity, Acc diagnostic accuracy.

Patients on beta-blocker therapy, who were responders during adenosine echocardiography testing (6 out of 9) showed functional improvement after revascularisation at follow-up. In these 9 patients the WMSI was $1.55 \pm 0.25$ at baseline, $1.35 \pm 0.27$ at peak adenosine $(\mathrm{p}<0.01)$, and $1.36 \pm 0.34(\mathrm{p}<0.01)$ at follow-up. In this subset, the diagnostic accuracy of adenosine for predicting viability was $88 \%$.

\section{Discussion}

Results of our study have shown that low dose adenosine stress echocardiography testing may identify potentially reversible myocardial dysfunction with high diagnostic confidence and feasibility.

It has been shown that revascularization of viable segments correlates with enhancement of regional and global myocardial function [22]. This is in agreement with our finding that wall motion score index indeed improved in revascularized patients at follow-up.

Nevertheless, we observed also spontaneous recovery of the segments in medically treated patients, but in less extent than was predicted by adenosine echocardiography, and wall motion score index at follow up in this group of patients did not improve.

Infusion of low dose adenosine has shown superior hemodynamic and safety profile. 
The possible physiologic basis for improvement in thickening and contractility of dyssynergic myocardial segments during low dose adenosine infusion could be the consequence of the following mechanisms:

1) Increase in blood flow within the ventricular wall that leads to myofiber stretching and an increase in contractile force through the Gregg phenomenon [23];

2) Functional improvement of dyssynergic segments during increased coronary flow states that are related to adenosine effect on microvasculature, recruitment of adjacent capillary beds especially in the endocardium and improved oxygen supply consumption ratios;

3) Cardioprotective effects of adenosine on glucose metabolism that tends to decrease cellular acidosis and Ca ++ overload, which may be associated with beneficial effects on mechanical function [24].

In addition, Rovai et al.[25] using dipyridamole contrast echocardiography, have shown that myocardial perfusion in the asynergic ventricular walls could be either preserved at baseline or recruitable by vasodilation. The primary effect of dipyridamole is adenosine A2-receptor mediated vasodilation on the coronary arteriole smooth muscle cell, leading to an increase in flow [20]. Our data are consistent with experimental and clinical studies $[12,13,15]$, showing that even very low doses of adenosine can evoke contractile response in asynergic but viable segments.

\section{Relation to Previous Studies}

To our knowledge, there are a limited number of studies in regard to adenosine echocardiography in detection of viable myocardium. Our study is the first one that used incremental low doses of adenosine in order to obtain maximal functional response and to avoid possible ishemia, that could be provoked with high adenosine doses. Our intention was to design protocol with minimum side effects and maximum diagnostic potentials, which could be safely used in everyday clinical practice, in patients with bad ventricular performance and in ordinary echocardiography lab. There are published data with higher adenosine doses (140 up to $180 \mathrm{mcg} / \mathrm{kg} / \mathrm{min}$ ) safely used in cath lab to assess the coronary flow reserve using intracoronary Doppler flow-wire, but this data are related mainly to the patients with myocardial infarction but with good ventricles $[26,27]$. The fact that test is performed in the cath lab diminishes the possibility of undesirable serious adverse events.

This protocol may have advantage over dobutamine in the patients with severe left ventricular dysfunction. Proarrhythmogenic effect of dobutamine is well known and it has been reported that occurrence of tachyarrhythmias during dobutamine stress testing are predicted by the extent of the left ventricular dysfunction but not by the presence or the extent of coronary artery disease [28].

One of the advantages of adenosine over dipyridamole and dobutamine is its short time to peak action allowing short duration of the test and its short half-life in circulation so there is no need for antidote.

Kranidis and al. [29] reported moderate sensitivity, specificity, positive and negative predictive value (54\%, 86\%, $65 \%$ and $80 \%$, respectively), of adenosine stress echocardiography in detecting viable myocardium in 52 patients with previous myocardial infarction. In their study they have used only deterioration of segmental motion as a sign of viability during infusion of $140 \mathrm{mcg} / \mathrm{kg} / \mathrm{min}$ of adenosine. This ischemia-based approach may miss the detection of viable, albeit not ischemic segments.

Case et al. [30] also reported the ability of adenosine echocardiography to detect viable myocardial segments in patients following thrombolytic therapy. They compared adenosine-induced changes in myocardial function in the infarct related territory with thallium scintigraphy criteria for viability. Out of 12 patients with evidence of viable myocardium by thallium scintigraphy criteria, only three patients demonstrated an improvement in regional function during adenosine infusion( $140 \mu \mathrm{g} / \mathrm{kg} / \mathrm{min})$, and 9 had ischemic response. Actually, regional function deteriorated in patients with high grade $(95 \pm 2 \%)$ stenosis and improved in those with nonflow limiting stenosis $(66 \pm$ $25 \%, p=0.03$ ). This is in keeping with observation of the previous study [29], that ischemic dose of adenosine (140 $\mu \mathrm{g} / \mathrm{kg} / \mathrm{min}$ ) would more likely provoke immediately deterioration of function and ischemia, rather than biphasic response - hallmark of viability, that could be seen with our proposed low dose stepwise infusion of adenosine.

Our data are comparable with results of Varga et al. [12] using infra-low dose dipyridamole $(0.28 \mathrm{mg} / \mathrm{kg})$ stress echocardiography for viability detection, reporting sensitivity and specificity of $78 \%$ and $94 \%$, respectively. They compared these results with results achieved with lowdose dobutamine stress echocardiography (up to $10 \mu \mathrm{g} /$ $\mathrm{kg} / \mathrm{min}$ ) in the same group of patients, yielding the sensitivity of $76 \%$ and specificity of $94 \%$, with a concordance of the methods of $93 \%$. Also, searching for the superior study protocol, Picano et al [13] have suggested the combined low dose dipyridamole - low dose dobutamine stress echocardiography, reporting increase in sensitivity from $67 \%$ (low dose dipyridamole) to $94 \%$ (combined low dose dipyridamole-dobutamine), with a slight and nonsignificant decrease in specificity (95\% to $92 \%)$. 
Sicari et al. [31] also used low dipyridamole echocardiography to assess the prognostic significance of myocardial viability in patient with severe left ventricular ischemic dysfunction. For the viability presence they have used cutoff value of delta wall motion score index $=0.20$ that corresponds on average to four segments showing viability, which is equivalent to $25 \%$ of the ventricle. The presence of myocardial viability at low dipyridamole echo test was the only independent predictor exerting protective effect on survival in revascularized patients.

\section{Study limitations}

The results we obtained with a new proposed protocol should be confirmed in the large scale multicenter study.

As the test result was not taken into account for decision making for revascularization, our study reflects the real life clinical routine. That explains why not every patient positive for viability underwent revascularization procedure.

The study population included patients with mild to moderate reduction of left ventricular performance; average wall motion score index was $1.55 \pm 0.30$. Our new protocol with incremental subischemic doses of adenosine is designed for "bad" ventricles, but in our clinical opinion it should be tested first for safety reasons and ability to provoke functional response in patients with less severe reduction of ventricular function. Nevertheless, this protocol may have advantage by avoiding provocation of ischemia that could be seen with higher adenosine doses, and rhythm disturbances that could be seen with dobutamine infusion.

In our study there are no comparative data with dipyridamole and dobutamine.

According to our results accuracy of adenosine testing is not influenced by beta-blocker therapy and this could be recommendation for its use in everyday clinical practice when it is not possible to rule out therapy for safety reasons.

The echocardiographically documented improvement of wall motion at follow-up was used as the definite method for evaluating the accuracy of stress-induced functional improvement, that may, per se, represent methodological limitation, since independent standards (fluorodeoxyglucose by positron emission tomography or thallium uptake by scintigraphy) were not utilized. The interpretation of digitally acquired echocardiography images was subjective and semiquantitive. However, in our study two independent observers reviewed all the studies, with low interobserver variability. When disagreement occurred regarding presence of viable segments, a third observer reviewed the study and his judgment was accepted.

\section{Clinical Implications}

In the present study we have shown that low dose adenosine stress echocardiography test has high diagnostic potential and feasibility in the group of patients with mild to moderate left ventricular impairment. Good effectiveness should justify higher adenosine price compared to dobutamine and dipyridamole. The study protocol and efficacy of low dose adenosine stress echocardiography should be assessed in larger number of patients with severe left ventricular dysfunction in whom the clinical question regarding the extent of viable tissue is most important.

The presence of myocardial viability can guide the clinical cardiologist towards more appropriate treatment by adding more information to conventional ones such as ejection fraction, coronary anatomy and inducible ischemia

Low dose adenosine stress echocardiography test, with excellent hemodynamic and safety profile, may be good alternative to low dose dobutamine and dipyridamole stress echocardiography testing for evaluation of myocardial viability.

\section{References}

I. Lee KS, Marwick TH, Cook SA, Go TR, Fix JS, James KR, Sapp SK, Maclntyre WJ and Thomas JD: Prognosis of patients wit left ventricular dysfunction, wit and without viable myocardium after myocardial infarction. Relative efficacy of medical therapy and revascularization Circulation 1994, 90:2687-2694.

2. DiCarli MF, Davidson M, Little R, Khanna S, Mody FV, Brunken RC, Czernin J, Rokhsar S, Stevenson LW and Laks H: Value of metabolic imaging with positron emission tomography for evaluating prognosis in patients with coronary artery disease and left ventricular dysfunction Am J Cardiol 1994, 73:527-533.

3. Gibson RS, Watson DD, Taylor G, Crosby IK, Wellons HL, Holt ND and Beller GA: Prospective assessment of regional myocardial perfusion before and after coronary revascularization surgery by quantitative thallium-20 I scintigraphy J Am Coll Cardiol 1983, I:804-815.

4. Neinaber CA, Brunken RC, Sherman CT, Yeatman LA, Gambhir SS, Krivokapich J, Demer LL, Ratib O, Chilad JS and Phelps ME: Metabolic and functional recovery of ischemic human myocardium after coronary angioplasty J Am Coll Cardiol 199I, I 8:966978.

5. Dilsizian $\vee$ and Bonow RO: Current diagnostic techniques of assessing myocardial viability in patients with hibernating and stunned myocardium Circulation 1993, 87: I-20.

6. Perrone-Filardi P, Pace L, Prastaro M, Squame F, Betocchi S, Soricelli A, Piscione $F$, Indolfi $C$, Crisci $T$, Salvatore $M$ and Chiariello $M$ : Assessment of myocardial viability in patients with chronic coronary artery disease: rest-4 hour-24 hour ${ }^{201}$ TI tomography versus dobutamine echocardiography Circulation 1996, 94:27|2-27|9.

7. Yoshida K and Lance Gould K: Quantitative relation of myocardial infarct size and myocardial viability by positron emission tomography to left ventricular ejection fraction and 3-year mortality with and without revascularization J Am Coll Cardiol 1993, 22:984-97.

8. Hata T, Nohara R, Fujita M, Hosokawa R, Lee L, Kudo T, Tadamura E, Tamaki N, Konishi J and Sasayama S: Noninvasive assessment of myocardial viability by positron emission tomography with IIC Acetate in patients with old myocardial infarction. 
Usefulness of low-dose dobutamine infusion Circulation 1996, 94:|834-|84|.

9. Nagueh SF, Vaduganathan P, Ali N, Blaustein A, Verani MS, Winters WL and Zoghbi WA: Identification of hibernating myocardium: Comparative accuracy of myocardial contrast echocardiography, rest-redistribution Thallium-20I tomography and dobutamine echocardiography J Am Coll Cardiol 1997, 29:985-93.

10. Vanoverschelde JL, Marwick TH and Gerber BL: Head to head comparison between exercise redistribution reinjection thallium single photon emission tomography and low dose dobutamine echocardiography for prediction of reversibility of chronic left ventricular ischemic dysfunction J Am Coll Cardiol 1996, 28:432-442.

II. Pasquet A, Lauer MS, Williams MJ, Secknus M-A, Lytle B and Marwick $\mathrm{TH}$ : Prediction of global left ventricular function after bypass surgery in patients with severe left ventricular dysfunction. Impact of pre-operative myocardial function, perfusion, and metabolism Eur Heart J 2000, 2 I: I 25- I 36.

12. Varga A, Ostojic M, Djordjevic-Dikic A, Sicari R, Pingitore A, Nedeljkovic I and Picano E.: Infra-low dose dipyridamole test. A novel dose regimen for selective assessment of myocardial viability by vasodilator stress echocardiography Eur Heart J 1996, 17:629-634.

13. Picano E, Ostojic M, Varga A, Sicari R, Djordjevic-Dikic A, Nedeljkovic $I$ and Torres M: Combined low dose dipyridamole-dobutamine stress echocardiography to identify myocardial viability J Am Coll Cardiol 1996, 27: $1422-$ | 428.

14. Mercier JC, Lando $V$ and Kanmatsuse K: Divergent effects of inotropic stimulation on the ischemic and severely depressed reperfused myocardium Circulation 1982, 66:397-400.

15. Stahl LD, Aversano TR and Becker LC: Selective enhancement of function of stunned myocardium by increased flow Circulation 1986, 74:843-5I.

16. Berne RM: The role of adenosine in the regulation of coronary blood flow Circ Res 1980, 47:807-8I3.

17. Belardinelli L, Linden J and Robert MR: The cardiac effects of adenosine Prog Cardiovasc Dis 1989, 32:73-97.

18. Cronstein BN, Daguma L, Nichols D, Hutchinson AJ and Williams M: The adenosine/neutrophil paradox resolved: Human neutrophils possess both $A I$ and $A 2$ receptors that promote chemotaxis and inhibit $\mathrm{O}_{2}$ generation, respectively $j$ Clin Invest 1990, 85: II50-II57.

19. Cronestein BN, Levin RI, Belanoff J, Weissmann G and Hirshhorn R: Adenosine: an endogenous inhibitor of neutrophil-mediated injury to endotelial cells / Clin Invest 1986, 78:760-770.

20. Ely SW and Berne RM: Protective effects of adenosine in myocardial ischemia Circulation 1992, 85:893-904.

21. American Society of Echocardiography Committee on Standards, Subcommittee on Quantitation of Two-Dimensional Echocardiograms, Schiller NB, Shah PM and Crawford M: Recommendation for quantitation of the left ventricle by two-dimensional echocardiography J Am Soc Echocardiogr 1989, 2:358-67.

22. Perrone-Filardi P, Pace L, Prastaro M, Piscione F, Betocchi S, Squame F, Vezzuto P, Soricelli a, Indolfi C, Salvatore M and Chiarello M: Dobutamine echocardiography predicts functional improvement of hypoperfused dysfunctional myocardium following revascularization in patients with coronary artery disease Circulation 1995, 91:2556-2565.

23. Gregg DE: Effect of coronary perfusion pressure or coronary flow on oxygen usage of the myocardium Circ Res 1963, 13:497500 .

24. Finegan BA, Lopaschuk GD, Coulson CS and Clanachan AS: Adenosine alters glucose use during ischemia and reperfusion in insolated rat hearts Circulation 1993, 87:900-908.

25. Rovai D, Zanchi M, Lombardi M, Magagnini E, Chella P, Pieroni A, Picano E, Ferdeghini M, Morris H, Distante A and L'Abbate A: Residual myocardial perfusion in reversibly damaged myocardium by dipyridamole contras echocardiography Eur Heart J 1996, 17:296-301.

26. De Bruyne B, Hersbach F, Pijls HJN, Bartunek J, Bech J-W, Heyndrickx RG and Gould KL: Abnormal epicardial coronary resistence in patients with diffuse atherosclerosis but normal coronary angiography Circulation 200I, 104:240I-2406.

27. De Bruyne B, Pijls HJN, Barbato E, Bartunek J, Bech J-W, Wijns W and Heyndrickx RG: Intracoronary and intravenous adenosine 5'triphosphate, adenosine, papaverine, and contrast medium to assess fractional flow reserve in humans Circulation 2003, 107:1877-| 883 .

28. Elhendy A, van Domburg RT, Bax JJ and Roelandt JR: Relation between the extent of coronary artery disease and tachyarrhythmias during dobutamine stress echocardiography $\mathrm{Am} J$ Cardiol 1999, 83:832-5.

29. Kranidis A, Bouki T, Kostopoulos K, Anthopoulos P, Kappos K, Antonellis J, Bonou M, Sideris A, Ralli D, Tavernarakis A, Kesse M and Anthopoulos L: Stress echocardiography using adenosine combined with nitroglycerin-dobutamine in the detection of viable myocardium in patients with previous myocardial infarction Angiology 1997, 48: I 27-I 33.

30. Case AR, Buckmire R, McLauglin DP and Keller M: Physiological assessment of coronary artery disease and myocardial viability in ischemic syndromes using adenosine echocardiography Echocardiography 1994, I I:133-143.

31. Sicari R, Ripoli A, Picano E, Borges AC, Varga A, Mathias W, Cortigiani L, Bigi R, Heyman J, Polimeno $S$ and on behalf of the VIDA (Viability Identification with Dipyridamole Administration) Study Group: The prognostic value of myocardial viability recognized by low dose dipyridamole echocardiography in the patients with chronic ichhaemic left ventricular dysfunction Eur Heart J 200 I, 22:837-844.
Publish with Bio Med Central and every scientist can read your work free of charge

"BioMed Central will be the most significant development for disseminating the results of biomedical research in our lifetime. "

Sir Paul Nurse, Cancer Research UK

Your research papers will be:

- available free of charge to the entire biomedical community

- peer reviewed and published immediately upon acceptance

- cited in PubMed and archived on PubMed Central

- yours - you keep the copyright

Submit your manuscript here:

http://www.biomedcentral.com/info/publishing_adv.asp 